08,04

\title{
Моноклинные центры редкоземельных $S$-ионов в кристаллах ортоалюмината иттрия
}

\author{
(C) В.А. Важенин ${ }^{1}$, А.П. Потапов ${ }^{1}$, Г.Р. Асатрян ${ }^{2}$, А.Г. Петросян ${ }^{3}$, К.Л. Ованесян ${ }^{3}$, А.В. Фокин ${ }^{1}$ \\ ${ }^{1}$ Институт естественных наук и математики Уральского феедерального университета, \\ Екатеринбург, Россия \\ ${ }^{2}$ Физико-технический институт им. А.Ф. Иофффе РАН, \\ Санкт-Петербург, Россия \\ ${ }^{3}$ Институт физических исследований НАН Армении, \\ Аштарак, Армения \\ E-mail: Vladimir.Vazhenin@urfu.ru
}

(Поступила в Редакцию 27 фревраля 2017 г.)

В кристаллах $\mathrm{YAlO}_{3}$, легированных европием и цирконием, исследован спектр ЭПР моноклинных центров $\mathrm{Gd}^{3+}$ и $\mathrm{Eu}^{2+}$, замещающих ионы иттрия. Определены параметры тонкой структуры указанных центров. Для центров с изотопом ${ }^{151} \mathrm{Eu}$ определены параметры сверхтонкого взаимодействия, обсуждается сверхтонкая структура центров с изотопом ${ }^{153} \mathrm{Eu}$.

Работа выполнена в рамках государственного задания Минобрнауки РФ для Уральского федерального университета и гранта Госкомитета по науке Армении 15RF-003. Измерения проведены на спектрометре Центра коллективного пользования „Современные нанотехнологии“ Уральского федерального университета.

DOI: 10.21883/FTT.2017.09.44852.052

\section{1. Введение}

Кристаллы ортоалюмината иттрия $\left(\mathrm{YAlO}_{3}\right)$, как и иттрий-алюминиевого граната $\left(\mathrm{Y}_{3} \mathrm{Al}_{5} \mathrm{O}_{12}\right)$, активированные редкоземельными элементами, являются хорошими лазерными материалами и активно используются в квантовой электронике. Возможно одновременное легирование $\mathrm{YAlO}_{3}$ редкоземельными ионами и ионами группы переходных металлов большой концентрации, заметно расширяющее свойства материала. Кроме того, кристаллы ортоалюмината иттрия, легированные ионами $\mathrm{Ce}^{3+}$ и $\mathrm{Eu}^{2+}$, оказались замечательными материалами для сцинтилляторов, применяемых в качестве детекторов ионизирующих излучений в астрофизике, медицинской аппаратуре и физике высоких энергий. Для оптимизации параметров этих материалов необходима информация о природе и структуре дефектов в реальных кристаллах, которая может быть получена с помощью магнитного резонанса.

Энергетическая структура основного состояния примесных центров $\mathrm{Cr}^{3+}[1,2], \mathrm{Fe}^{3+}[3], \mathrm{Gd}^{3+}[3-5], \mathrm{Ce}^{3+}$, $\mathrm{Nd}^{3+}, \mathrm{Er}^{3+}[6], \mathrm{Tm}^{3+}$ [7] в $\mathrm{YAlO}_{3}$ и их атомная структура (в частности, локализация) уже были исследованы методом электронного парамагнитного резонанса (ЭПР). Настоящая работа представляет магниторезонансное исследование в кристаллах алюмината иттрия высокоспиновых центров редкоземельных $S$-ионов $\mathrm{Gd}^{3+}$ и $\mathrm{Eu}^{2+}$.

\section{2. Методика эксперимента}

В работе исследованы монокристаллы алюмината иттрия с европием $\left(\mathrm{YAlO}_{3}: \mathrm{Eu}\right)$, выращенные методом вер- тикальной направленной кристаллизации $[8,9]$ с использованием высокочистых исходных компонентов-оксидов и молибденовых контейнеров $\left(T_{m}\left[\mathrm{YAlO}_{3}\right]=1916^{\circ} \mathrm{C}\right)$. Для стабилизации в кристаллической решетке достаточных количеств центров $\mathrm{Eu}^{2+}$ в расплав дополнительно вводилась четырехвалентная примесь $\left(\mathrm{Zr}^{4+}\right)$ в виде оксида $\mathrm{ZrO}_{2}$, кристаллизация проводилась в сильно восстановительной атмосфере $\left(\mathrm{Ar} / \mathrm{H}_{2}\right)$. Образец, отобранный для исследований, был получен из расплава, соответствующего по составу $\mathrm{Y}_{1-x-y} \mathrm{Eu}_{x} \mathrm{Zr}_{y} \mathrm{AlO}_{3} \quad(x=0.02, y=0.002)$, и имел желтоватый оттенок. В приближении участия всех ионов $\mathrm{Zr}^{4+}$ в стимулировании валентных переходов $\mathrm{Eu}^{3+} \rightarrow \mathrm{Eu}^{2+}$ концентрация $\mathrm{Eu}^{2+}$ в образце составляла около 0.2 at\%. Как показали результаты исследований, изложенные далее, в образце в виде неконтролируемых примесей присутствовали также ионы $\mathrm{Cr}^{3+}$ и $\mathrm{Gd}^{3+}$.

Измерения спектров ЭПР неориентированных образцов проводились при комнатной температуре на спектрометре трехсантиметрового диапазона EMX Plus Bruker. Ориентация образцов в резонаторе спектрометра производилась с помощью штатного одноосного автоматического гониометра и приспособления, позволяющего вращать образец в вертикальной плоскости. Для достижения выделенных ориентаций $(\mathbf{B} \| \mathbf{a}, \mathbf{b}, \mathbf{c}$, где $\mathbf{B}-$ индукция магнитного поля, $\mathbf{a}, \mathbf{b}, \mathbf{c}-$ кристаллографические направления) использовались угловые зависимости положений переходов наблюдаемых в кристалле центров $\mathrm{Cr}^{3+}$ и $\mathrm{Gd}^{3+}$, полученные авторами [1-3]. 


\section{3. Результаты эксперимента и обсуждение}

Кристаллы $\mathrm{YAlO}_{3}$ имеют структуру искаженного перовскита с пространственной группой $P b n m\left(D_{2 h}^{16}\right)$ и параметрами элементарной ячейки $a=5.176 \AA$, $b=5.332 \AA, c=7.356 \AA$. Четыре позиции иттрия попарно связаны операцией инверсии и отражением в плоскостях $c a$ и $c b$. Примесные редкоземельные ионы $\mathrm{Gd}^{3+}$ и $\mathrm{Eu}^{2+}$ (электронные спины $S=7 / 2$ ) в этом кристалле естественно замещают „квазиредкоземельные“ ионы иттрия в позициях, имеющих единственную операцию симметрии - отражение в плоскости $a b$ (группа точечной симметрии $C_{S}$ ). В случае нелокальной зарядовой компенсации примесного иона будут существовать два магнитно-неэквивалентных центра $\mathrm{Eu}^{2+}\left(\right.$ а также $\left.\mathrm{Gd}^{3+}\right)$, которые становятся эквивалентными только в плоскостях $a c$ и $b c$.

Как уже отмечалось выше, кроме переходов моноклинных центров $\mathrm{Eu}^{2+}$ в исследуемых образцах наблюдались сигналы центров $\mathrm{Cr}^{3+}$ и $\mathrm{Gd}^{3+}$ (рис. 1,2). Добиваясь путем вращения образца слияния сигналов от магнитнонеэквивалентных центров $\mathrm{Cr}^{3+}, \mathrm{Gd}^{3+}$ и $\mathrm{Eu}^{2+}$, мы получили спектры при $\mathbf{B}\|\mathbf{a}, \mathbf{B}\| \mathbf{b}$. Для получения ориентации B $\| \mathbf{c}$ необходимо было достичь наряду со слиянием неэквивалентных спектров еще и экстремальности положений сигналов. Кроме того, была измерена угловая зависимость вида спектра при вращении магнитного поля в плоскости, близкой к $a b$, с шагом $5^{\circ}$ (рис. 3 ), а затем $2^{\circ}$.

3.1. $\mathrm{YAlO}_{3}: \mathrm{Gd}^{3+}$. Для подтверждения адекватности соотнесения полученных ориентаций и кристаллографических направлений была проведена оптимизация параметров спинового гамильтониана с учетом экспериментальных положений переходов центров $\mathrm{Gd}^{3+}$. Моноклинный спиновый гамильтониан в определении [10] в системе координат $\mathbf{x}\|\mathbf{a}, \mathbf{y}\| \mathbf{b}, \mathbf{z} \| \mathbf{c}$ имеет следующий вид:

$$
\begin{aligned}
H_{\text {sp }} & =g \beta(\mathbf{B S})+1 / 3\left(b_{20} O_{20}+b_{22} O_{22}+c_{22} \Omega_{22}\right) \\
& +1 / 60\left(b_{40} O_{40}+b_{42} O_{42}+c_{42} \Omega_{42}+b_{44} O_{44}+c_{44} \Omega_{44}\right) \\
& +1 / 1260 \sum_{m} b_{6 m} O_{6 m},
\end{aligned}
$$

где $g-g$-фактор, $\beta$ - магнетон Бора, $O_{n m}$ и $\Omega_{n m}-$ косинусоидальные и синусоидальные спиновые операторы Стивенса [10], $b_{n m}$ и $c_{n m}$ - параметры тонкой структуры. В используемой системе координат параметры тонкой структуры двух магнитно-неэквивалентных центров $\mathrm{Gd}^{3+}$ отличаются знаками $c_{n m}$. Полученные нами величины параметров представлены в табл. 1, где также приведены результаты авторов [3-5]. Параметры шестого ранга (последнее слагаемое в выражении (1)) оказались очень малыми и поэтому не приводятся.

Отметим, что не приведенные в табл. 1 величины $b_{4 m}$ из [3] были на три порядка меньше значений $b_{2 m}$, а система координат повернута вокруг оси $z$ в плоскости $a b$

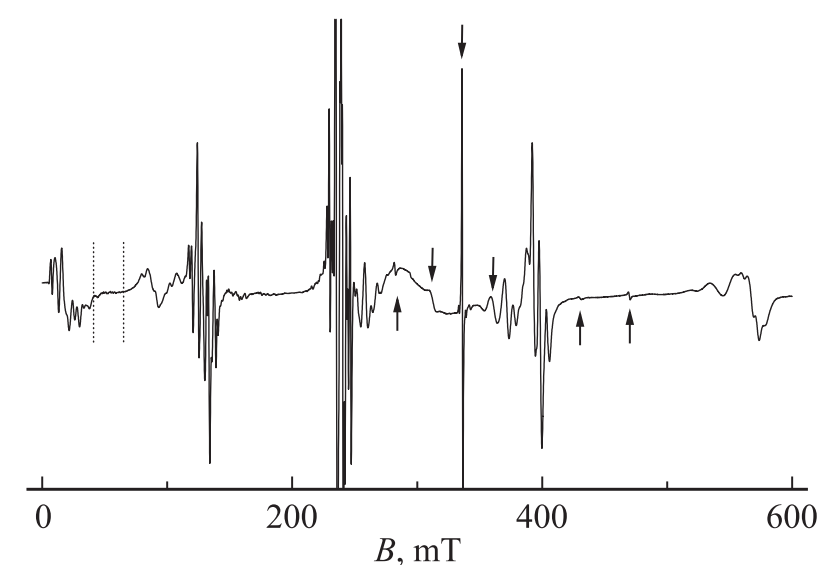

Рис. 1. Спектр ЭПР (производная сигналов поглощения) $\mathrm{YAlO}_{3}: \mathrm{Eu}, \mathrm{Zr}$ при В $\|$ а на частоте $9448 \mathrm{MHz}$. Верхние стрелки показывают сигналы $\mathrm{Cr}^{3+}$, нижние $-\mathrm{Gd}^{3+}$. Область спектра, выделенная пунктирными линиями, приведена на рис. $7, b$.

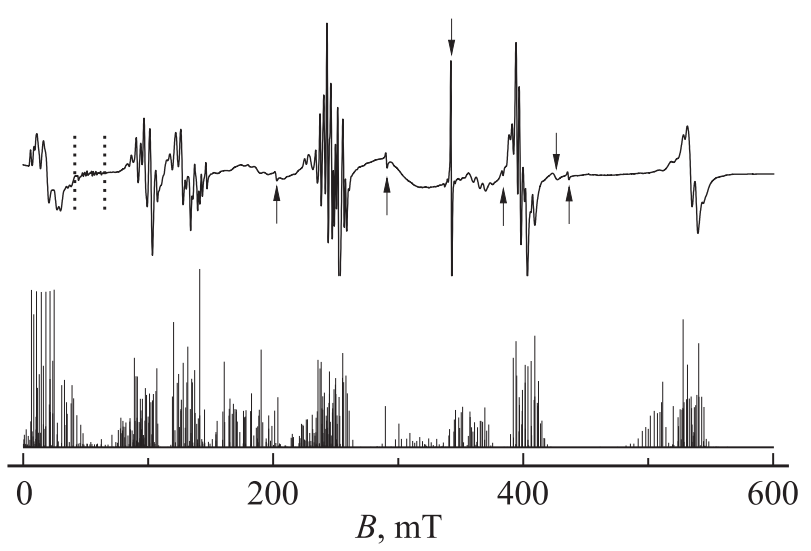

Рис. 2. Спектр ЭПР $\mathrm{YAlO}_{3}: \mathrm{Eu}, \mathrm{Zr}$ при $\mathbf{B} \| \mathbf{b}$ на частоте $9449 \mathrm{MHz}$ и расчетная сверхтонкая структура для изотопа ${ }^{151} \mathrm{Eu}$. Верхние стрелки показывают сигналы $\mathrm{Cr}^{3+}$, нижние $-\mathrm{Gd}^{3+}$. Область спектра, выделенная вертикальными пунктирными линиями, приведена на рис. 7,a.

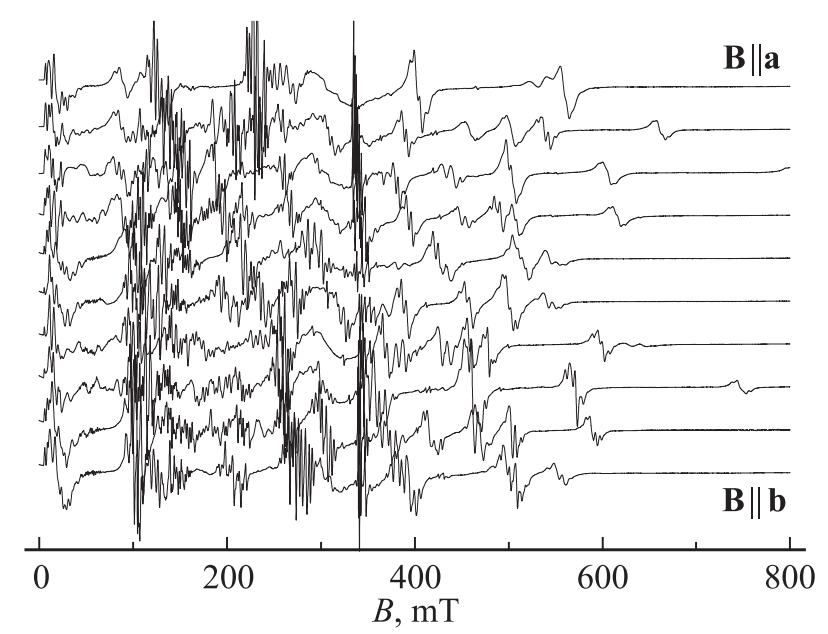

Рис. 3. Ориентационное поведение спектра $\mathrm{YAlO}_{3}: \mathrm{Eu}, \mathrm{Zr}$ при вращении магнитного поля в плоскости, близкой к $a b$. 
на угол $\Delta \varphi=(1 / 2) \arctan \left(c_{22} / b_{22}\right)=28^{\circ}$, в результате чего параметр $c_{22}$ обращается в нуль, т.е. тензор второго ранга спинового гамильтониана приобретает более симметричную ромбическую структуру. Величины $\Delta \varphi$, соответствующие параметрам, полученным нами и авторами $[4,5]$, также приведены в табл. 1.

Следует заметить, что при повороте системы координат на угол, обнуляющий значение $c_{22}$, параметры $c_{4 m}$ и $c_{6 m}$ не исчезают, и, следовательно, спиновый гамильтониан остается моноклинным. Величина $\left(b_{22}^{2}+c_{22}^{2}\right)^{0.5}$ при поворотах системы координат вокруг оси $z$ остается постоянной и в работах, упомянутых в табл. 1 , практически не различается. Аналогичные отклонения магнитной оси низкоспиновых редкоземельных ионов от оси $a$ в плоскости $a b$ получены авторами [6,7]: 41.4 для $\mathrm{Er}^{3+}$, $30.5^{\circ}$ для $\mathrm{Nd}^{3+}, 31.8^{\circ}$ для $\mathrm{Ce}^{3+}$ и $55^{\circ}$ для $\mathrm{Tm}^{3+}$.

На рис. 4 сплошными кривыми показано ориентационное поведение положений переходов одного из магнитно-неэквивалентных центров $\mathrm{Gd}^{3+}$, соответствующее параметрам из табл. 1. Угловые зависимости для второго центра, параметры которого отличаются знаками $c_{n m}$, показаны штриховыми кривыми. Небольшое число экспериментальных точек на рис. 4 в ориентациях магнитного поля, отличных от В $\|$ a и $\mathbf{B} \| \mathbf{b}$, обусловлено, как видно из рис. $1-3$, сложностью регистрации слабых сигналов $\mathrm{Gd}^{3+}$ на фоне интенсивных сигналов $\mathrm{Eu}^{2+}$.

Согласие полученных нами параметров и угловых зависимостей с результатами $[4,5]$ свидетельствует о правильной идентификации спектров с направлениями в кристалле и, следовательно, о возможности использо-

Таблица 1. Параметры спинового гамильтониана центров $\mathrm{Gd}^{3+}$ в $\mathrm{YAlO}_{3}$ в системе координат $\mathbf{x}\|\mathbf{a}, \mathbf{y}\| \mathbf{b}, \mathbf{z} \| \mathbf{c}$ (среднеквадратичное отклонение расчетных частот от экспериментальных $F(N)$, где $N$ - число использованных экспериментальных положений сигналов, и параметры $b_{n m}, c_{n m}$ приведены в $\mathrm{MHz}, \Delta \varphi-$ угол поворота системы координат, приводящего к обнулению $c_{22}$ )

\begin{tabular}{c|c|c|c}
\hline \multirow{2}{*}{ Параметры } & \multicolumn{3}{|c}{ Лит. ссылка } \\
\cline { 2 - 4 } & {$[4,5]$} & {$[3]^{*}$} & Наст. раб. \\
\hline$g$ & 1.992 & & 1.991 \\
$b_{20}$ & $-334.8^{* *}$ & 278.7 & $-245^{* *}$ \\
$b_{22}$ & 1621.4 & -2926.0 & 1805 \\
$c_{22}$ & 2432.2 & 0 & 2367 \\
$b_{40}$ & 19.0 & & 34 \\
$b_{42}$ & -69.0 & & -227 \\
$c_{42}$ & -170.1 & & -17 \\
$b_{44}$ & -123.3 & & -163 \\
$c_{44}$ & 99.9 & & 146 \\
$\left(b_{22}^{2}+c_{22}^{2}\right)^{0.5}$ & 2923 & 2926 & 2976 \\
$F(N)$ & $5(100)$ & & $43(17)$ \\
$\Delta \varphi$, deg & 28 & 28 & 26.5
\end{tabular}

Примечан ие. * Система координат повернута вокруг оси $z$ на $28^{\circ}$. ** Знак $b_{20}$ не определялся.

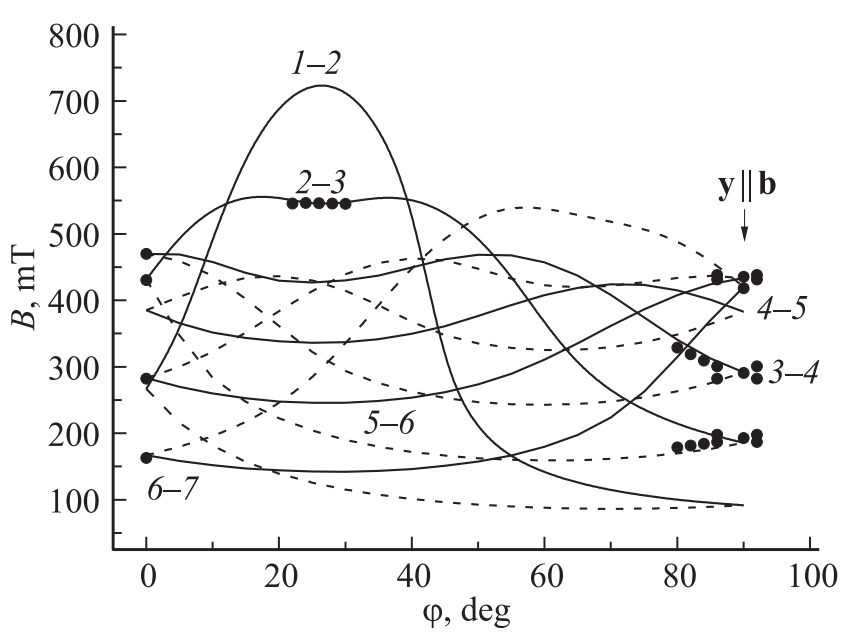

Рис. 4. Угловая зависимость положений переходов моноклинных центров $\mathrm{Gd}^{3+}$ вблизи плоскости $a b$. Сплошные кривые расчет с параметрами из табл. 1, штриховые - расчет с $c_{n m}$, имеющими противоположные знаки, точки - эксперимент. Цифры около кривых - номера уровней, между которыми происходят соответствующие переходы (нумерация снизу).

вания экспериментальных спектров в ориентациях $\mathbf{B} \| \mathbf{a}$, $\mathbf{B}\|\mathbf{b}, \mathbf{B}\| \mathbf{c}$ для определения параметров, описывающих неизвестный ранее центр $\mathrm{Eu}^{2+}$ в неориентированных кристаллах алюмината иттрия.

3.2. $\mathrm{YAlO}_{3}: \mathrm{Eu}^{2+}$. Как видно из рис. 1-3, сигналы центров $\mathrm{Eu}^{2+}$ (ядерные спины изотопов ${ }^{151} \mathrm{Eu},{ }^{153} \mathrm{Eu}$ равны $5 / 2$, естественная распространенность 48 и 52\% соответственно) характеризуются различной формой линии. Среди них можно выделить сигналы с довольно разрешенной, но несимметричной сверхтонкой структурой (СТC), широкие и практически бесструктурные, а также сигналы промежуточной формы.

Похожая ситуация для центров $\mathrm{Eu}^{2+}$ имеет место в иттрий-алюминиевом [11] и лютеций-алюминиевом [12] гранатах. Представленный на рис. 2 оценочный расчет СТС для изотопа ${ }^{151} \mathrm{Eu}$ с константой сверхтонкого взаимодействия $A \approx-100 \mathrm{MHz}[11,12]$ и параметрами тонкой структуры, определенными далее, однозначно свидетельствует о том, что разная форма сигналов в спектре центров $\mathrm{Eu}^{2+}$ в $\mathrm{YAlO}_{3}$ обусловлена в первую очередь различной сверхтонкой структурой (количество компонент, их интенсивности и взаимное расположение) на разных электронных переходах. Необходимость учета СТС от изотопа ${ }^{153} \mathrm{Eu}$ еще больше затрудняет возможность соотнесения расчетных и экспериментальных положений электронно-ядерных переходов и делает невозможным одновременное определение параметров спинового гамильтониана, описывающих тонкую и сверхтонкую структуры центров $\mathrm{Eu}^{2+}$ с использованием матрицы энергии 48 порядка $((2 S+1)(2 I+1))$.

В связи с этим параметры тонкой структуры определялись с использованием матрицы энергии восьмого порядка для гипотетического изотопа $\mathrm{Eu}^{2+}$ с нулевым 
ядерным спином, за резонансные положения которого на разных электронных переходах брались значения магнитного поля, приближенно соответствующие центрам СТС. Для описания спектра использовалась система координат $\mathbf{X}\|\mathbf{a}, \mathbf{Y}\| \mathbf{c}, \mathbf{Z} \| \mathbf{b}$. В этой системе спиновый гамильтониан тонкой структуры имеет вид

$$
\begin{aligned}
H_{\mathrm{sp}} & =g \beta(\mathbf{B S})+1 / 3\left(b_{20} O_{20}+b_{21} O_{21}+b_{22} O_{22}\right) \\
& +1 / 60\left(b_{40} O_{40}+b_{41} O_{41}+b_{42} O_{42}+b_{43} O_{43}+b_{44} O_{44}\right) \\
& +1 / 1260 \sum_{m} b_{6 m} O_{6 m}
\end{aligned}
$$

где обозначения величин такие же, как в (1), но параметры тонкой структуры двух магнитно-неэквивалентных центров $\mathrm{Eu}^{2+}$ различаются знаками $b_{n m}$ с нечетными проекциями $m$. Результат оптимизации методом наименьших квадратов параметров (2) с использованием 22 резонансных положений при $\mathbf{B}\|\mathbf{a}, \mathbf{B}\| \mathbf{b}, \mathbf{B} \| \mathbf{c}$ приведен в табл. 2. Среднеквадратичное отклонение экспериментальных частот от расчетных $F=45 \mathrm{MHz}$, что довольно велико, но легко объясняется используемым способом определения резонансных магнитных полей для переходов гипотетического изотопа $\mathrm{Eu}^{2+}$. Параметры шестого ранга порядка погрешности определения и поэтому не приводятся.

Для подтверждения адекватности параметров гамильтониана (2) проведено сравнение экспериментальных угловых зависимостей положений переходов гипотетического изотопа $\mathrm{Eu}^{2+}$ с расчетными (рис. 5). Обнаружено, что спектры при $\mathbf{B} \| \mathbf{b}$ хорошо согласуются, тогда как при В $\|$ a они могут быть объяснены лишь при допущении, что экспериментальная угловая зависимость на рис. 5 получена при $\varphi \approx 15^{\circ}$. В связи с этим полярная зависимость положений переходов (сплошные кривые на рис. 5) была рассчитана с параметрами из табл. 2 при $\varphi=15^{\circ}$.

Поворот системы координат на угол $\Delta \theta=20.6^{\circ}$ вокруг оси $Y$ приводит к обращению в нуль параметра

Таблица 2. Параметры спинового гамильтониана моноклинных центров $\mathrm{Eu}^{2+}\left({ }_{b n m}\right.$ в $\left.\mathrm{MHz}\right)$ в двух системах координат (вторая повернута вокруг $Y$ на угол $20.6^{\circ}$ ).

\begin{tabular}{c|c|c}
\hline Параметры & $\mathbf{X}\|\mathbf{a}, \mathbf{Y}\| \mathbf{c}, \mathbf{Z} \| \mathbf{b}$ & $\Delta \theta=20.6^{\circ}$ \\
\hline$g$ & 1.9905 & 1.9905 \\
$b_{20}$ & $1592^{*}$ & 2147 \\
$b_{21}$ & 5908 & 0 \\
$b_{22}$ & -1974 & 2529 \\
$b_{40}$ & 1 & -58 \\
$b_{41}$ & -324 & -229 \\
$b_{42}$ & -178 & -54 \\
$b_{43}$ & -384 & -236 \\
$b_{44}$ & -125 & -73
\end{tabular}

При ме ч ан ие. * Предполагается, что $b_{20}>0$.

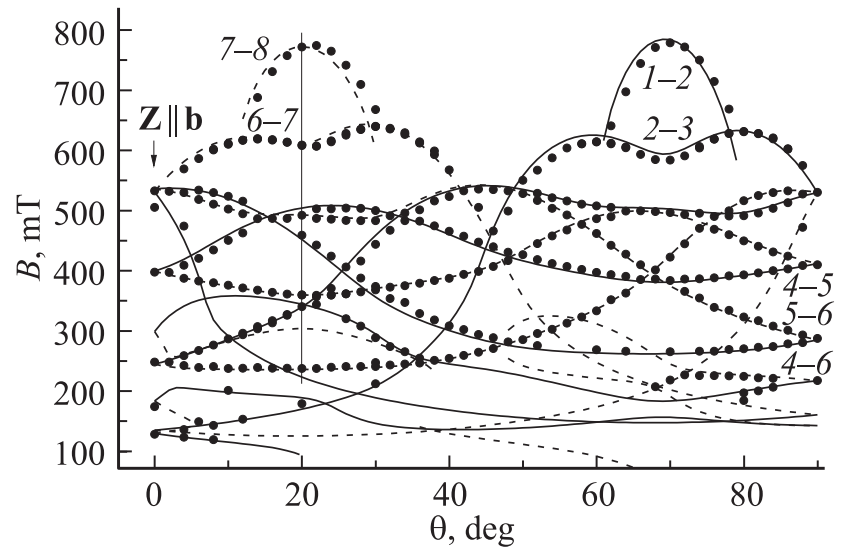

Рис. 5. Полярная зависимость положений переходов моноклинных центров $\mathrm{Eu}^{2+}$ при $\varphi=15^{\circ}$. Сплошные кривые расчет с параметрами из табл. 2, штриховые - расчет c $b_{n m}$ противоположного знака (при нечетном $m$ ), точки эксперимент.

тонкой структуры $b_{21}$, отвечающего за отличие моноклинного тензора второго ранга от ромбического; величины остальных параметров после поворота приведены в табл. 2. На рис. 5 указанный поворот соответствует сдвигу оси $Z$ в позицию, помеченную вертикальной прямой. Как видно, даже для высокополевых переходов угловые зависимости не совсем симметричны относительно новой оси $Z$, для низкополевых переходов асимметрия еще больше. Следовательно, центры $\mathrm{Eu}^{2+}$ после поворота остаются моноклинными, при этом их отличие от ромбической симметрии заметно больше, чем у центров $\mathrm{Gd}^{3+}$. Следует заметить, что определенные здесь и в работах [3-7] величины поворота системы координат, приводящего к уменьшению числа параметров тонкой структуры, невозможно просто связать со структурой окружения редкоземельного иона [3].

Сложная СТС, наблюдаемая даже в ориентациях магнитного поля вдоль кристаллографических осей $a, b, c$ (рис. 1-3), очень затрудняет определение параметров, описывающих сверхтонкую структуру изотопов ${ }^{151} \mathrm{Eu}$ и ${ }^{153} \mathrm{Eu}$. Наиболее простая структура (шесть эквидистантных линий примерно равной интенсивности) для изотопов европия может наблюдаться на переходах между практически чистыми состояниями. Как показали оценочные расчеты СТС для изотопа ${ }^{151} \mathrm{Eu}$ (рис. 2), таких переходов в ориентации В $\|$ b всего два. На рис. 6, где показаны все переходы для гипотетического изотопа $\mathrm{Eu}$, они выделены жирными отрезками. Но переход в уровнях 5-7 (нумерация снизу) практически совпадает по полю с переходом в уровнях 4-6, в связи с чем идентификация электронно-ядерных переходов и оценка величины сверхтонкого взаимодействия изотопа ${ }^{151} \mathrm{Eu}^{2+}$ при $\mathbf{B}\|\mathbf{b}\| \mathbf{Z}$ оказались возможными лишь на переходе 7-8 в поле $\sim 530 \mathrm{mT}$ (рис. 6). Переход 7-8- это сильно запрещенный переход (с $\Delta M \approx 7$, где $M$ - проекция электронного спина), поэтому его интенсивность много 


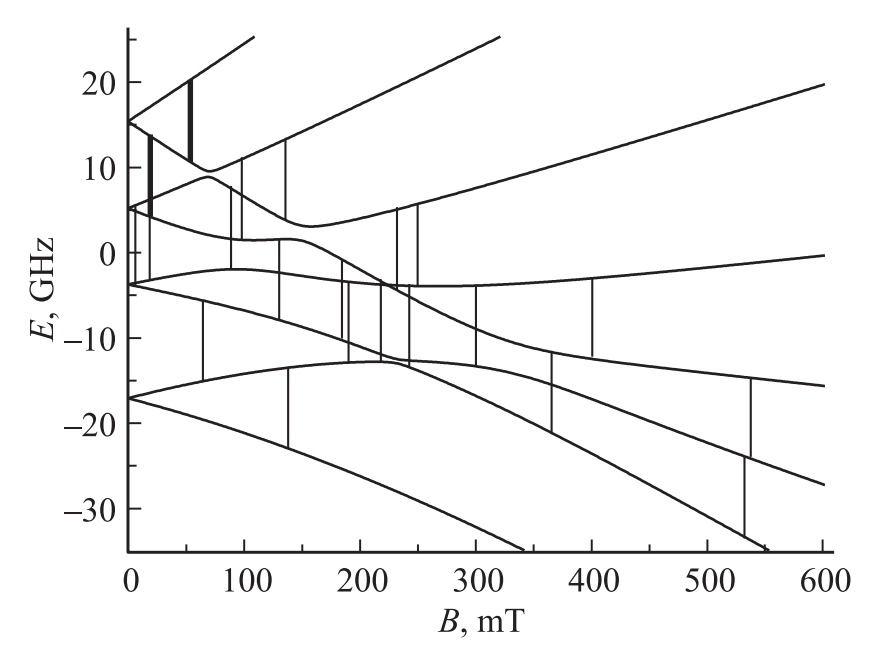

Рис. 6. Уровни энергии и ЭПР-переходы для гипотетического изотопа $\mathrm{Eu}$ с нулевым ядерным спином при $\mathbf{B}\|\mathbf{b}\| \mathbf{Z}$ на частоте $9449 \mathrm{MHz}$.
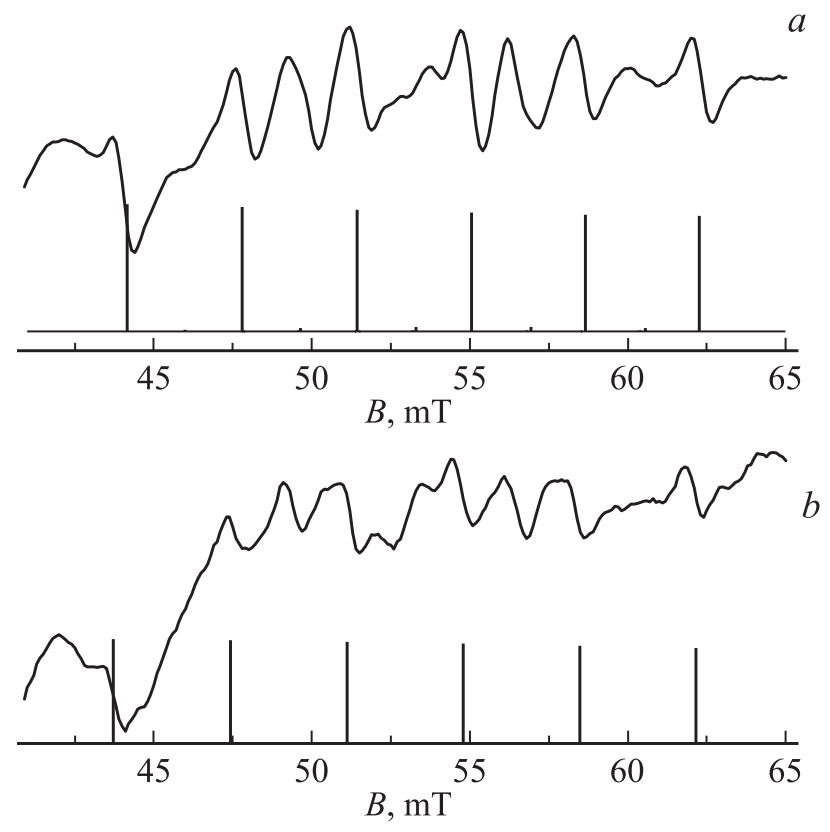

Рис. 7. Экспериментальная и расчетная СТС для изотопа ${ }^{151} \mathrm{Eu} . \quad a-$ для перехода 7-8 при $\mathbf{B} \| \mathbf{b}\left(\theta=0^{\circ}, \varphi=0^{\circ}\right)$ на частоте $9449 \mathrm{MHz}, b-$ для перехода $1-2$ при $\mathbf{B} \| \mathbf{a}\left(\theta=90^{\circ}\right.$, $\varphi=0^{\circ}$ ) на частоте $9448 \mathrm{MHz}$.

меньше (рис. 2), чем у большинства других переходов в этой ориентации. Выделенная пунктирными линиями область спектра рис. 2 показана на рис. 7, $a$.

При $\mathbf{B} \| \mathbf{a}\left(\theta=90^{\circ}, \varphi=0^{\circ}\right)$ идентификация электронно-ядерных переходов ${ }^{151} \mathrm{Eu}$ оказалась возможной тоже только на одном электронном переходе. Это также запрещенный переход с $\Delta M \approx 7$ еще меньшей интенсивности, но только в уровнях $1-2$, регистрируемый в том же магнитном поле (выделенная область спектра на рис. 1), что и переход в уровнях 7-8 при $\mathbf{B} \| \mathbf{b}\left(\theta=0^{\circ}\right.$, $\left.\varphi=0^{\circ}\right)$. Близость резонансных положений переходов
1-2 и 7-8 в двух ориентациях обусловлена тем, что в случае $b_{22} \approx b_{20}$ при повороте магнитного поля на $90^{\circ}$ происходит опрокидывание системы уровней энергии. Такой эффект наблюдался в [11]. Сверхтонкая структура перехода 1-2 при В || а представлена на рис. 7, $b$.

В общем случае для описания СТС изотопов $\mathrm{Eu}^{2+}$ необходимо кроме сверхтонкого учесть еще и квадрупольное взаимодействие, описываемое параметром $Q$. Его влияние заключается в нарушении эквидистантности интенсивных линий СТС и появлении слабых компонент в промежутках между основными. Сходство СТС ионов ${ }^{151} \mathrm{Eu}$ (рис. 7, $a$ и $b$ ) на указанных выше переходах в ориентациях $\mathbf{B}\|\mathbf{b}\| \mathbf{Z}$ и $\mathbf{B}\|\mathbf{a}\| \mathbf{X}$ (шесть эквидистантных линий) дает основание утверждать, что константа $Q$ для ${ }^{151} \mathrm{Eu}$ много меньше константы сверхтонкого взаимодействия и может не учитываться. Численной минимизацией среднеквадратичного отклонения расчетных частот от экспериментальных для двенадцати значений магнитного поля (рис. 7, $a, b)$ с использованием матрицы энергии 48 порядка получена величина $A=-95(3) \mathrm{MHz}$. Знак константы $A$ выбран по аналогии c $[11,12]$. Влияние константы квадрупольного взаимодействия $Q$ на СТС переходов, использованных для оценки константы $A$, должно проявляться начиная со значения $\pm(20-30) \mathrm{MHz}$.

$\mathrm{У}$ изотопа ${ }^{153} \mathrm{Eu}$ ядерный магнитный момент примерно в 2.2 раза меньше, чем у ${ }^{151} \mathrm{Eu}$, поэтому в простейших случаях шесть линий СТC ${ }^{153} \mathrm{Eu}$ должны располагаться между четырьмя средними компонентами СТC ${ }^{151} \mathrm{Eu}$. Зато квадрупольный момент ${ }^{153} \mathrm{Eu} \mathrm{в} \sim 2.5$ раза больше, чем у ${ }^{151} \mathrm{Eu}$. C учетом оценочных значений константы квадрупольного взаимодействия для ${ }^{151} \mathrm{Eu}$ получаем величину отношения $A / Q \approx 1$ для ${ }^{153} \mathrm{Eu} \mathrm{в} \mathrm{YAlO}_{3}$. При таком соотношении расчетная структура должна состоять из полутора десятков неэквидистантных и соизмеримых по интенсивности компонент, которые частично перекрываются с переходами СТC ${ }^{151} \mathrm{Eu}$, что делает практически невозможным ее соотнесение с экспериментальным спектром (рис. 7, $a, b)$.

По-видимому, точное определение параметров, описывающих СТC $\mathrm{Eu}^{2+}$ в алюминате иттрия, будет возможно только в кристаллах $\mathrm{YAlO}_{3}$, обогащенных изотопами ${ }^{153} \mathrm{Eu}$ или ${ }^{151} \mathrm{Eu}$.

\section{4. Заключение}

В неориентированных монокристаллах алюмината иттрия, легированного европием и цирконием, при комнатной температуре исследован спектр парамагнитного резонанса моноклинных центров $\mathrm{Gd}^{3+}$ (неконтролируемая примесь) и $\mathrm{Eu}^{2+}$, замещающих ионы иттрия с симметрией $C_{S}$. Путем сравнения наблюдаемых спектров $\mathrm{Gd}^{3+} \mathrm{c}$ результатами коллег из Казанского университета установлены кристаллические оси образцов. Определены параметры тонкой структуры центров $\mathrm{Gd}^{3+}$ и $\mathrm{Eu}^{2+}$, 
для центров $\mathrm{Eu}^{2+}$ осуществляется нелокальная компенсация заряда. Для центров европия с изотопом ${ }^{151} \mathrm{Eu}$ определены параметры сверхтонкого взаимодействия, обсуждается СТС центров с изотопом ${ }^{153} \mathrm{Eu}$.

\section{Список литературы}

[1] A. Pinto, N.Z. Sherman. J. Magn. Res. 6, 422 (1972).

[2] M. Yamaga, H. Takeuchi, T.P.J. Han, B. Henderson. J. Phys.: Condens. Matter 5, 8097 (1993).

[3] R.L. White, G.F. Herrmann, J.W. Carson, M. Mandel. Phys. Rev. A 136, 231 (1964).

[4] Н.М. Низамутдинов, Н.М. Хасанова, А.А. Галеев, Г.Р. Булка, В.М. Винокуров, В.А. Аккерман, Г.А. Ермаков. Кристаллография 34, 893 (1989).

[5] Н.М. Хасанова, Н.М. Низамутдинов, Г.Р. Булка, В.М. Винокуров, В.А. Аккерман, Г.А. Ермаков, А.А. Маркелов. Физика минералов и их синтетических аналогов. Изд-во Казан. ун-та, Казань (1988). С. 73.

[6] Г.Р. Асатрян, J. Rosa. ФТT 44, 830 (2002).

[7] Г.Р. Асатрян, А.П. Скворцов, Г.С. Шакуров. ФТТ 55, 958 (2013).

[8] А.А. Чернов, Е.И. Гиваргизов, Х.С. Багдасаров, В.А. Кузнецов, Л.Н. Демьянец, А.Н. Лобачев. Современная кристаллография. Наука, М. (1980). 337 с.

[9] A.G. Petrosyan, G.O. Shirinyan, K.L. Ovanesyan, C. Pedrini, C. Dujardin. J. Cryst. Growth 198/199, 492 (1999).

[10] С.А. Альтшулер, Б.М. Козырев. Электронный парамагнитный резонанс соединений элементов промежуточных групп. Наука, М. (1972). С. 121.

[11] В.А. Важенин, А.П. Потапов, Г.Р. Асатрян, А.Г. Петросян, К.Л. Ованесян, А.В. Фокин, Г.С. Шакуров. ФТТ 58, 2406 (2016).

[12] В.А. Важенин, А.П. Потапов, Г.Р. Асатрян, А.Г. Петросян, А.В. Фокин, М.Ю. Артёмов. ФТТ 59, 1323 (2017). 